\title{
Satisfacción laboral como indicador de desarrollo profesional en universidades
}

\author{
Elvis Eliana Pinto Aragón \\ Universidad de la Guajira \\ Colombia \\ Alain Castro Alfaro \\ Corporación Universitaria Rafael Núñez \\ Colombia \\ Annherys Isabel Paz Marcano \\ Universidad de la Guajira \\ Colombia
}




\title{
Satisfacción laboral como indicador de desarrollo profesional en universidades
}

\author{
Elvis Eliana Pinto Aragón ${ }^{1 D}$, Alain Castro Alfaro ${ }^{2}$ iD y Annherys Isabel Paz Marcano $^{1}$ iD \\ 1 Universidad de la Guajira, Colombia \\ ${ }^{2}$ Corporación Universitaria Rafael Núñez, Colombia
}

Para citaciones: Pinto, E., Castro, A., Paz, A. (2020). Satisfacción laboral como indicador de desarrollo profesional en las universidades. Panorama Económico, 28(1), 2340. https://doi.org/10.32997/pe-2020-2667

Recibido: 30/09/2019

Aprobado: 10/12/2019

Autor de correspondencia:

Elvis Eliana Pinto Aragón

epinto@uniguajiira.edu.co

Proyecto de investigación: Análisis de la calidad de vida laboral de los empleados de carrera administrativa de la Universidad de La Guajira (Colombia).

Editor: Andrés Escobar Espinoza Universidad de Cartagena, Colombia

Tipología IBN Publindex:

Artículo de investigación científica y tecnológica.

Copyright: (C) 2020. Pinto, E., Castro, A., $\mathrm{Paz}, \mathrm{A}$. Este es un artículo de acceso abierto, distribuido bajo los términos de la licencia https://creativecommons.org/licenses/bync-sa/4.0/ la cual permite el uso sin restricciones, distribución y reproducción en cualquier medio, siempre y cuando que el original, el autor y la fuente sean acreditados.

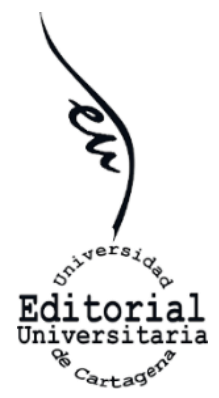

RESUMEN

El presente documento analiza la satisfacción laboral como indicador de desarrollo profesional entre los colaboradores en la Universidad de La Guajira (Colombia), el cual genera una sinergia que propicia el trabajo colaborativo en beneficio del alcance de los objetivos misionales. Metodológicamente, se trata de una investigación inscrita en un paradigma positivista, de tipo descriptivo y analítica, con un diseño no experimental, transaccional de campo. Para la recolección de información se aplicaron encuestas de 24 ítems con opción de respuestas múltiples cada una, a 59 empleados de carrera administrativa. El instrumento fue validado por 5 expertos y se obtuvo un Alfa de Cronbach del 91 \% de fiabilidad. Se concluye que la satisfacción laboral como indicador de desarrollo, se sustenta de factores extrínsecos a la institución objeto de estudio, y que promueve la sinergia, convivencia y bienestar entre los colaboradores favoreciendo el desarrollo profesional en las áreas de trabajo. Asimismo, se sugiere mantener el factor de satisfacción en los espacios de trabajo, consiguiendo incrementar la motivación en el desempeño laboral de los colaboradores de carrera administrativa.

Palabras clave: Satisfacción laboral; desarrollo profesional; universidades.

JEL: M10, M12, M54

\section{Job satisfaction as a career development indicator in higher education institutions}

\section{ABSTRACT}

This document analyzes the job satisfaction as an indicator of professional development among collaborators at the University of La Guajira (Colombia). Such indicator generates a synergy that promotes collaborative work aiming to the achievement of objectives included into the institutional mission. Methodologically, it is a research in harmony with the positivist paradigm, descriptive and analytical, with a non-experimental, transactional field design. For the information-gathering, surveys of 24 items with multiple choice options were applied to 59 administrative career employees. The instrument was validated by 5 experts and a Cronbach's Alpha of 91\% reliability was obtained. It is concluded that job satisfaction as an indicator of development is based on factors external to the institution under study, and that it promotes synergy, coexistence and well-being among employees, promoting professional development in work areas. Likewise, it is suggested to maintain the satisfaction factor in working places, managing to increase the motivation in the work performance of the administrative career collaborators.

Keywords: Job satisfaction; Professional development; Higher Education Institutions. JEL: M10, M12, M54 


\section{INTRODUCCIÓN}

La globalización ha traído consigo ambientes de incertidumbre en los escenarios de trabajo, los cuales transcienden en la gestión de las organizaciones, sin exclusión de las universidades como institución de educación superior, donde los cambios y las transformaciones del entono las han inducido a unir esfuerzos por el establecimiento de estrategias que ofrezcan respuestas a las necesidades laborales de los colaboradores; entre ellas, la satisfacción de su gente, como agente que promueve la calidad de vida, la motivación y el desempeño hacia la excelencia y calidad de las actividades misionales de la institución. Esto quiere decir, acciones de gestión humana, las cuales propendan a la generación de paradigmas focalizados en garantizar el bienestar que contribuye a incrementar la competitividad, el desarrollo y crecimiento de su fuerza laboral. Según Harris, Franco y Paz (2014), las instituciones de educación superior están inmersas en múltiples desafíos del entorno globalizado, las cuáles las conducen a plantearse cambios en los sistemas de gestión, permitiendo actualizar los esquemas de trabajo, tanto administrativos como académicos, investigativos y de extensión, como estrategia de compromiso con el fin de generar conocimientos y el desarrollo de un talento humano calificado, con miras al servicio de una educación que se base en los principios misionales de calidad y excelencia educativa.

En este sentido, las universidades están llamadas a cumplir con su rol académico, de extensión e investigación, como también ser agente portador de desarrollo de su fuerza laboral, para conseguir la excelencia en el desempeño de los procesos de trabajo, permitiendo agregar valor a los indicadores que dan respuestas a las actividades consagradas en los planes institucionales mediante una mística laboral que las hagan merecedoras de un slogan de academia y proyección social inserta en los pilares que enaltecen sus principios misionales, de la mano con el talento humano, el cual es el motor que dinamiza los objetivos de la academia. Según Pinto, Brito y Mendoza (2016), las universidades en sus planes de desarrollo, deben incorporar proyectos y planes de talento humano como estrategia de fortalecimiento de las competencias laborales de sus empleados, donde su desempeño contribuya a la excelencia organizacional.

En este particular, refieren Núñez, Paz, Salón \& Rosales (2012), que la dinámica de las universidades en la última década, ha replanteado las funciones misionales de docencia, investigación y extensión hacia un compromiso compartido con su gente y el entorno. Siendo así, como agentes de formación de personas, deben incluir en sus planes actividades que coadyuven, tanto al bienestar como el desempeño laboral de su fuerza laboral, considerando las actitudes, competencias y conocimientos, los cuales intervienen en los resultados y la excelencia de las actividades misionales. Por tanto, la satisfacción laboral, constituye un tema de interés en la gestión del talento humano en las instituciones, donde no están ajenas las universidades y, en particular, la Universidad de La Guajira, siendo los factores extrínsecos un indicador del desarrollo de las personas, ya que le genera estabilidad laboral, siendo reflejado en la jornada de trabajo, uso de medios de comunicación, retribución, ambiente, beneficios y servicios alcanzados por la relación institución-colaboradores, por tratarse de algunos aspectos, que contribuyen a la satisfacción, motivación y el rendimiento laboral de las personas, los cuales vienen a ser una consecuencia entre la contribución que realiza el trabajador con su expectativa del entorno. A su vez, de acuerdo con Pinto, Brito y Mendoza (2016), la universidad de 
La Guajira debe establecer patrones estratégicos, a fin de satisfacer las necesidades de los colaboradores, siendo un factor fundamental del éxito de los procesos de trabajo e integración de ellos para conseguir la excelencia institucional.

En la primera parte se presenta la revisión de literatura, posteriormente se detalla la metodología y los principales resultados. Finalmente, se presentan las principales conclusiones.

\section{REVISION DE LITERATURA}

Cabe destacar que son diversos los estudios desarrollados acerca de la gente, pero pocos conocen los factores externos asociados a la satisfacción en los espacios laborales (Hernández, 2017; Castro, 2017). Pues la satisfacción laboral, se convierte en el nutriente que alimenta el interior de las personas, colocándose de manifiesto en el desempeño de su rol de trabajo. Según Pérez y Azzollini (2013), la satisfacción laboral, es un tema tratado en los últimos años en el quehacer investigativo en distintos campos de estudios científicos, donde se hace mención a su asociatividad a variables de importancia en la vida organizacional, tales como: la productividad y el rendimiento, el estrés, el burnout, el ausentismo, la rotación entre otros (Briceño et al., 2019).

Por otro lado, se infiere el estudio realizados por Marquina (2013) donde admite que la satisfacción laboral y el compromiso organizacional son dos constructos de interés en los estudios planteados por psicólogos, en el área de Recursos Humanos. Por tal motivo, la investigación indagó ambos constructos y su relación en los empleados de una universidad privada en el año 2011. Para conocer el grado de satisfacción de las personas de la institución, se empleó la Escala de Satisfacción Laboral SL - SPC (Palma, 1999) y la Escala del Grado de Compromiso de los Empleados Universitarios ASD (Grajales, 2000) a 121 personas pertenecientes a una población de 300 individuos en condición de empleados. Los resultados de la investigación, mostraron la presencia de insatisfacción laboral en un 51.3\%, de los colaboradores consultados, para medir el comportamiento de la variable satisfacción laboral con las dimensiones condiciones laborales/materiales y políticas administrativas de la institución objeto de estudio; no obstante, a pesar del registro del dato precedente, el 91\% ratifica altos niveles de compromiso organizacional, por lo que se sienten identificados con la institución, ofreciendo lealtad. En cuanto al resultado de la correlación entre la variable satisfacción y compromiso laboral, se empleó la fórmula de Pearson logrando un 0.73, el cual es estadísticamente significativa ( $p=0.10)$ en la población estudiada.

Son diversas las teorías que han abordado la satisfacción de las personas en las áreas de trabajo, centrando la atención en los resultados para lograr la productividad deseada en términos económicos para su estabilidad en el mercado, dejando en segundo plano la satisfacción de este en el ambiente, donde ejerce sus funciones. Es de esta manera, como se puede hacer mención a la teoría de Herzberg citado de Rodríguez, Núñez \& Cáceres, (2010), el cual también, se denomina como teoría de los dos factores, donde el primer factor se refiere a los aspectos intrínseco, derivado de la relación empleado-trabajo, realización, reconocimiento, la promoción, el trabajo estimulante y la responsabilidad, y el segundo factor el extrínseco, suscrito en las políticas institucionales, relaciones interpersonales, sueldo, supervisión y las condiciones de trabajo; entre otros. 
En este sentido, se explica que la referida teoría ha sido la fuente básica, para estudiar la satisfacción de las personas en sus áreas laborales, donde a modo de interés en la investigación, se sustenta de preceptos, para conocer la realidad percibida por el personal objeto de estudio, donde no solo se traduce en bienestar, sino al igual en la satisfacción en el lugar de trabajo. Cabe mencionar, que la satisfacción laboral comprende la percepción adquirida de los colaboradores en el desempeño de su trabajo, siendo un indicador para lograr sus propósitos no solo personales sino también profesionales, de allí que se conjugue tanto en sentimientos de las personas como las emociones. De tal manera, que se realiza un recorrido conceptual para conocer la postura que argumentan diversos autores, en cuanto a la satisfacción laboral. Es decir, se trata de la actitud ante el trabajo, resaltando discrepancias acerca de concepción asociada a la función laboral asignada versus los resultados que demanda su desempeño, razón por la que suele percibirse de manera positiva o negativa. De allí que a continuación se muestra, la conceptualización de autores como:

Tabla 1. Satisfacción Laboral

\begin{tabular}{|c|c|c|}
\hline Autores & Año & Conceptualización \\
\hline \multirow{2}{*}{$\begin{array}{l}\text { Kinicki \&Kreitner, citado } \\
\text { en Canton y Tellez } \\
\text { Chiang, Martín y Núñez }\end{array}$} & 2016 & $\begin{array}{c}\text { Es una respuesta afectiva o emocional generada por el individuo en su } \\
\text { ambiente de trabajo. }\end{array}$ \\
\hline & 2011 & $\begin{array}{c}\text { Situación que experimenta ante su trabajo y los diferentes aspectos del } \\
\text { mismo. }\end{array}$ \\
\hline Ivancevich & 2009 & Es una actitud de los individuos en sus funciones laborales. \\
\hline Davis \& Newstrom & 2008 & $\begin{array}{l}\text { Conjunto de respuestas afectivas que una persona manifiesta acerca } \\
\text { de su trabajo }\end{array}$ \\
\hline
\end{tabular}

Fuente: Elaboración propia.

Es así, como se percibe de acuerdo con las posturas de los autores consultados que la satisfacción laboral, se sustenta al interior de las personas en su comportamiento, es decir cuando la persona se sienta eficaz o no con el resultado con su desempeño, condicionado además de factores externos, generados por las interacciones en el espacio de trabajo y el entorno laboral. Para Harris, Franco y Paz (2014) el empleador, deben promover en sus colaboradores lugares de satisfacción, motivación, pertenencia, responsabilidad, logro, progreso, reconocimiento, condiciones sociales, compromiso, intereses en el desempeño laboral, ya que conducen al éxito de las universidades en el mercado, además del incremento de la productividad y calidad institucional.

De tal manera que puede agregarse que un trabajo emblemático de la administración para aportar en el quehacer del hombre en el trabajo (Herzberg et al., 1959; Warr, 1987 citado de Rodríguez, Núñez y Cáceres, 2010) en su aporte a la gestión organizacional esta las características intrínsecas del trabajo en el área operacional propio del desempeño del trabajo mismo, entre ellos: oportunidad de control, uso de habilidades, metas y ambiente de trabajo. En este mismo orden de ideas, el referido autor agrega a las características extrínsecas del trabajo sumidas en el desempeño tanto de las tareas como las actividades y no solo sustentadas en la descripción de su contenido, entre ellas: la disponibilidad de dinero, seguridad física, oportunidad de relaciones interpersonales y el enfoque social. Por consiguiente, se trata de la dimensión que estudia los elementos e indicadores asociados a la satisfacción de la persona, bajo el contenido de su trabajo, los cuales inducen a crear oportunidades de desarrollo en la organización, tales como: reconocimientos, logros, características del trabajo, supervisión, políticas de sueldos y salarios, relaciones interpersonales y políticas de la empresa, entre otras; cabe mencionar, 
que la estructura de políticas de desarrollo y oportunidades en una empresa se establece en las instituciones desde la gestión del talento humano, los cuales difiere entre las públicas y privadas; y en algunos casos del tipo de trabajo desempeñado en ellas.

Koontz et al. (2012) enlistan ciertos aspectos que consideran satisfactores, y por tanto motivadores, estando vinculados al ejercicio del contenido del trabajo, y se relaciona con: el logro, el reconocimiento, el trabajo desafiante, el avance y el crecimiento en el trabajo; de tal maneara que su presencia en el campo de trabajo determina el valor de sentimientos de satisfacción o no satisfacción o bien no insatisfacción. Es de este modo, se hace referencia a los indicadores de satisfacción laboral, como son:

a) Posibilidad de crecimiento. Es la probabilidad que tiene un individuo de lograr ascenso en su ambiente de trabajo (Robbins, 2012).

b) Reconocimiento. Para Gibson et al. (2013), el reconocimiento, se trata de del agradecimiento expresado por la administración ante un trabajo bien hecho.

Por otra parte, Bohlander et al. (2008), señalan que el reconocimiento, es una herramienta asociada a las necesidades personales como lo son el ego o autoestima, donde el individuo internaliza el campo de trabajo, siendo una recompensa para conseguir destacarse en su entorno laboral; al igual debe estar sustentado en la autorrealización y el respeto a sí mismo. De allí que, a modo general, se trata del aporte expresado por los gerentes a los colaboradores, al momento de observar los resultados alcanzados del desempeño en las actividades asignadas, es decir destacando sus competencias ante los trabajos bien realizados entre los demás.

a) Logro. resultados obtenidos por el esfuerzo realizado en la organización. Por su parte, de acuerdo a Ivancevich (2009), el logro es el deseo que siente una persona de alcanzar objetivos claros, autodeterminados y moderadamente complejos, con retroalimentación, basados en la consecución de los objetivos auto determinados.

b) Características del trabajo. Está referida con la satisfacción del empleado en relación a la naturaleza de la labor que realiza.

c) Supervisión. proceso mediante el cual se establece el control de la organización. Al respecto Chuder \& Sherman (2007), sostienen que el papel del supervisor en la institución es de vital importancia, donde todo lo que se haga depende principalmente de él, al hacerse responsable de la unidad, no obstante, el supervisor sirve de eslabón o de enlace e influye en su personal estimulándolo, promoviendo buenas relaciones y sirviendo de paradigma ante todos sus empleados.

Chiavenato (2011) expone que el salario es un elemento motivador, que sustenta la calidad de vida de un individuo en el contexto social, estando relacionado con el trabajo. En las organizaciones, el salario es indicador asociado tanto a un costo como a una inversión, por ello viene hacer el costo del producto o del servicio final e inversión, representando el dinero aplicado a un factor productivo (el trabajo) en un intento por obtener un rendimiento mayor al corto o mediano plazo. Agrega el autor, que las estructuras de salarios, deben están sustentadas a criterios equitativos y justos, teniendo en cuenta dos aspectos importantes, el primero, los salarios deben relacionarse con los 
puestos de la organización, a fin de tener un equilibrio interno de la estructura salariar de los colaboradores de ella; en segundo los salarios con respecto a empresas con naturaleza similar a ella en el mercado, a fin de tener una referencia de equilibrio externo en los salarios devengados por los colaboradores.

Para Castillo (2008) el salario, es la cantidad de dinero que pacta en el contrato de trabajo y es cancelado de manera ordinaria como resultado del trabajo al colaborador, sin la adición de otros elementos que lo integran. En este particular la legislación colombiana no consagra el significado de salario, no obstante, hace referencia en el (artículo 23 del Código Sustantivo del Trabajo Colombiano), como un elemento suscrito en el contrato de trabajo. Pero al artículo 127, modificado por el artículo 14 de la Ley 50 de 1990, se admite que el salario no solo se trata de un valor adquirido de una remuneración ordinaria, fija o variable, sino se trata de un bien recibido por un trabajador en dinero o en especie como contraprestación directa del servicio, sea cualquiera la forma o denominación que se adopte, como primas, sobresueldos, bonificaciones habituales, valor del trabajo suplementario o de las horas extras, valor del trabajo en días de descanso obligatorio, porcentajes sobre ventas y comisiones, entre otros aspectos.

a) Relaciones interpersonales. Davis y Newstron (2008), señalan que son las interacciones entre compañeros de trabajo o individuos que comparten actividades en una misma organización.

b) Políticas de la empresa. Davis y Newstron (2008), la definen como las conductas intencionadas diseñadas para incrementar o proteger la influencia e intereses personales de un individuo, en este caso cuando la institución considera a su personal, toma en cuenta como política la capacitación y el crecimiento tanto personal como profesional de sus empleados, para que obtengan el conocimiento y habilidades, desarrollando así sus actitudes en beneficio de su trabajo.

En este particular, los factores extrínsecos de satisfacción laboral en la Universidad de La Guajira, está vinculada a los elementos planteados desde la dirección de talento humano, están contenida en aspectos como: compensación monetaria, resultante del trato salarial justo para los empleados de carrera administrativa adscritos a ella, por tanto, consiste en la presencia de diversos indicadores como oportunidad y desarrollo, reconocimiento, logros, características del trabajo, supervisión, sueldos y salarios, relaciones interpersonales y políticas de la empresa, entre otros.

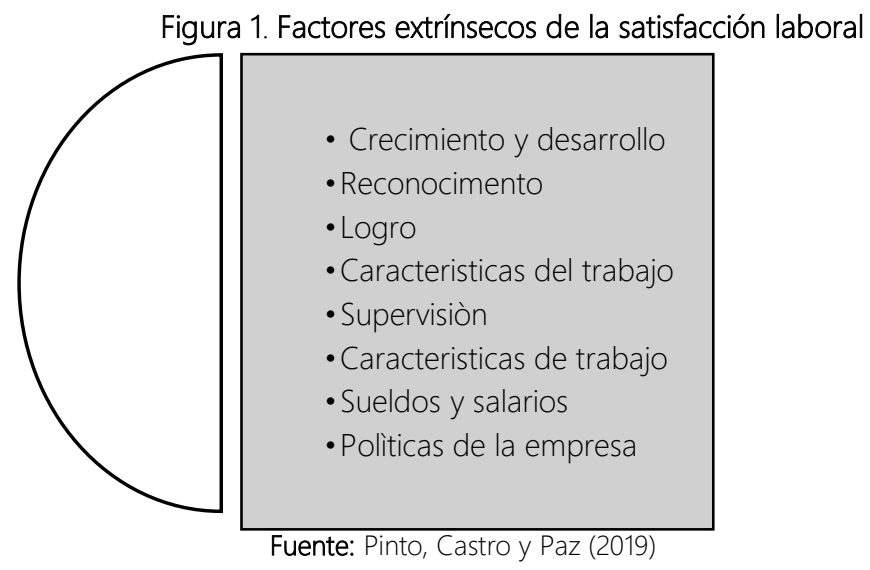




\section{METODOLOGIA}

La presente investigación, corresponde al paradigma positivista, teniendo como finalidad analizar la satisfacción laboral como indicador de desarrollo de los colaboradores en la Universidad de La Guajira, siendo los objetos de estudio para conocer las características referentes a hechos insertos en la variable, pudiendo mejorar el bienestar de los colaboradores, aunado a satisfacer sus necesidades. La investigación aplicada busca la generación de conocimiento con diligencia directa en situaciones de la sociedad o el sector productivo, teniendo como propósito encontrara hallazgos, ocupándose del proceso de enlace entre la teoría y el producto (Lozano, 2014). Asimismo, coincide Murillo (2008, citado en Vargas, 2009), la investigación aplicada, también se denomina práctica o empírica, sustentada en el criterio de aplicación o utilización de los conocimientos adquiridos, además de obtenerse otros, luego de implementar y sistematizar la experiencia basada en investigación desarrollada. En este sentido, la investigación, es de tipo aplicada, donde se ofrece a partir del bosquejo de conocimiento aportar respuesta a una situación en la universidad de la Guajira, los cuales quizás afectaría la satisfacción laboral de los colaboradores, como parte de su compromiso institucional por garantizar la calidad de vida de su personal entre ellos los empleados de carrera administrativa.

Por otra parte, se trata de una investigación de tipo descriptiva, el cual busca especificar las propiedades, características y perfiles importantes de personas, grupos, comunidades o cualquier otro fenómeno que se someta a un análisis (Hernández, et al, 2014; Méndez, 2013). Considerando los criterios de los autores citados, puede inferirse que se obtuvieron datos relacionados con el comportamiento de la variable satisfacción laboral en empleados de carrera administrativa de la Universidad de La Guajira Colombia, pudiendo lograr características específicas de la situación objeto de estudio. Por consiguiente, se trata de un estudio de campo, donde la información se extrajo de manera directa de los sujetos involucrados de la situación objeto de investigación, en este el escenario de trabajo administrativo de la universidad de La Guajira. (Arias. 2016) refiere que la investigación de campo, recolecta datos directamente en la realidad donde se generan los hechos, sin manipular o controlar variable alguna; del mismo modo coincide con (Tamayo y Tamayo, 2010), quien establece que los estudios de campo recogen datos directamente de la realidad.

En correspondencia al diseño de investigación, este trabajo se argumenta del no experimental, en el que no es manipulada la información a criterio del investigador, sino depende del comportamiento de la variable en el escenario de estudio, por lo tanto, el investigador suele ser un sujeto imparcial en la opinión emitida por las personas involucradas en ella. Para (Hernández, et al, 2014), las investigaciones no experimentales, llevan a cabo sin la manipulación deliberada de variables, donde solo se observan los fenómenos en su ambiente natural para después analizarlos. Asimismo, admite (Arias, 2016) que el diseño no experimental el investigador obtiene la información, pero no altera las condiciones existentes.

Por otro lado, se trata de un diseño no experimental de tipo transversal descriptiva, en el cual los datos del comportamiento de la variable, se obtuvo en un momento único seleccionado a juicio de los investigadores, sin invención de ellos para luego ser analizados e interpretados (Hernández et al., 2014) los diseños no experimentales de 
estudio transversales recolectan datos en un solo momento, en un tiempo único, teniendo objeto detallar variables y analizar su comportan miento en un lapso dado. Concordando de la misma manera con (Bernal, 2010) al admitir que la investigación seccional o transversal, consigue información del objeto de estudio (población o muestra) una única vez en un momento dado.

La población, según Bernal (2010), quien afirma que... "es el conjunto de todos los elementos a los cuales se refiere la investigación" (p. 160). Por otra parte, Hernández et al., (2014) refieren que se trata del conjunto de todos los casos que concuerdan con determinadas especificaciones, partiendo de características de contenido, de lugar y el tiempo. En este sentido, de acuerdo a la fuente conceptual de los autores citados, la población de estudio, se delimito al personal de carrera administrativa de la Universidad de La Guajira estando representada por 59 personas, caracterizadas como finita y accesible, razón por la que no se realizó un cálculo muestral, sino se estudió en su totalidad, constituyendo un censo poblacional (Tabla 2).

Tabla 2. Distribución de trabajadores, Universidad de La Guajira

\begin{tabular}{cc}
\hline Cargos & Números de Colaboradores \\
\hline Profesional & 28 \\
Técnicos & 15 \\
Auxiliares & 16 \\
\hline Total & 59 \\
\hline Fuente: Dirección de Talento humano de la Universidad de La Guajira (2017)
\end{tabular}

Según Arias (2016) en el censo poblacional, recaba información en la totalidad de la población. Coincide Méndez (2013) que un censo estudia todos los elementos involucrados en la población, siendo aconsejable aplicarla cuando es finita; asimismo cuando se quiere obtener información inmediata del estudio. En relación a la técnica de obtención de datos se utilizó la encuesta. De igual manera, Méndez (2013) señala que la encuesta es un instrumento de observación formado por una serie de preguntas formuladas por el investigador para ser contentada por el encuestado según las respuestas establecidas de manera objetiva e imparcial acerca del objeto de investigación. Para el registro de la información se utilizó un cuestionario, contentivo a un conjunto de ítems relacionado con el objeto de estudio, teniendo a modo de respuesta alternativas múltiples. Para Tamayo y Tamayo (2010), un cuestionario es un instrumento objeto de recolección de datos estructurados por un conjunto de reactivos los cuales pretenden evaluar los indicadores surgidos de la operacionalización del estudio. Por otro lado, para Arias (2016) un cuestionario, es un instrumento escrito formado por un conjunto de preguntas acerca de la variable a medir. En este sentido, el instrumento para medir los factores extrínsecos de la satisfacción laboral en la universidad objeto de estudio, se conformó por 24 reactivos redactados en forma de afirmaciones con opciones múltiples de respuesta de siempre, casi siempre, algunas veces, casi nunca y nunca.

En cuanto a la determinación de la eficacia del instrumento de recolección de datos diseñado por los investigadores para medir el comportamiento de la variable, fue evaluado por la experticia técnica y metodológica de 10 expertos, los cuales expresaron sus sugerencias para construir la versión final del cuestionario, ha aplicado a la población objeto de estudio. Para Hernández, et al. (2014), la validez es el grado donde un instrumento mide la variable que busca medir. Al igual, coincide con Arias (2016) que la 
validez del instrumento debe los ítems mostrar una redacción coherente con los objetivos de investigación. Posteriormente, la pertinencia del instrumento diseñado, para calcular el comportamiento de la variable en el escenario de estudio, se efectuó una prueba piloto con 10 sujetos de instituciones con características similares a la población, permitiendo obtener la confiabilidad. Para Hernández et al. (2014), la confiabilidad de un instrumento es el grado que un instrumento produce resultados consistentes y coherentes, es decir en que su aplicación repetida al mismo individuo u objeto produce resultados iguales. En la presente investigación, se acudió a la formula estadística de Alfa Cronbach alcanzado un índice de confiabilidad de 0.91 simbolizado un 91\% de fiabilidad.

Para Méndez (2013) las técnicas estadísticas, son empleadas para analizar e interpretar la información obtenida de la aplicación del instrumento de recolección de datos a los sujetos que hacen parte de la población, siendo representadas por el cálculo de índices de frecuencia, media aritmética, varianza, desviación estándar, coeficiente de variación, entre otros. Para efectuar el análisis e interpretación de los datos resultantes de la aplicación del cuestionario, se utilizó en el registro y organización de la información, la hoja de cálculo de Excel donde se tabularon los datos, permitiendo determinar los resultados. Previamente, se codifico la información, se fijó un código numérico a las respuestas contenidas en el cuestionario, siendo estas: siempre 5, casi siempre 4, a veces 3, casi nunca 2, nunca 1. La codificación para Hernández et al. (2014) consiste en asignar a los datos un valor numérico o símbolo que los represente para lograr el valor cuantitativo; para luego ser registrado en tablas, según Méndez (2013) la tabulación, se realiza para organizar la información, para ser procesada y cuantificada por ítems para facilitar la presentación en tablas.

En este particular, la organización de los datos de las respuestas derivadas de la aplicación del cuestionario, se registró en tablas para realizar el cálculo estadístico, con estimación de herramientas descriptivas de frecuencias absolutas y relativas e interpretada por un baremo. Según Hernández et al (2014), la estadística descriptiva, describe datos, los valores o las puntuaciones alcanzadas por variable, mientras la distribución de frecuencia, son puntuaciones ordenadas en categoría y generalmente se presentan como tabla.

En cuanto al procedimiento del trabajo de investigación, se inició con el desarrollo de un anteproyecto, detallando en su primera fase el planteamiento y formulación del problema, objetivos tanto generales como específicos; la justificación y delimitación de la investigación. Seguidamente, en la segunda fase, se mostraron los antecedentes, bases teóricas de autores que sustentan los criterios de la variable y el sistema de variable. En la tercera fase, se describe el campo metodológico, identificando el paradigma, tipo y diseño de investigación, población, materiales e instrumento de recolección de datos, validez y confiabilidad del instrumento; asimismo el análisis estadístico aplicado. En la cuarta fase se analizaron y discutieron los datos recogidos de la aplicación del instrumento, en este caso cuestionario a la población objeto. Finalmente, se presentan conclusiones, recomendaciones y referencias bibliográficas que sustentan el trabajo de investigación. Luego, a modo de presentación se elaboró el informe final, además del presente artículo como producto del quehacer investigativo realizado. 


\section{RESULTADOS}

El análisis de los resultados se llevó a cabo empleando la estadística descriptiva, determinado por el cálculo de distribución de frecuencias absolutas y relativas para conocer el comportamiento de la variable factores extrínsecos de satisfacción laboral, teniendo como escenario de investigación el área administrativa de la Universidad de La Guajira. En este sentido, se calcularon los valores promedios de los ítems de la dimensión e indicadores, siendo interpretados empleando un baremo de interpretación de datos promedios. Asimismo, la distribución de frecuencia permitió conocer el nivel porcentual de la opinión emitida por los sujetos encuestados; cabe mencionar que el cálculo de los datos frecuenciales de la variable, se muestra en tabla e ilustran en un gráfico de barras para la comprensión del lector.

Tabla 3. Baremo de interpretación de los niveles de satisfacción laboral

\begin{tabular}{cc}
\hline Rangos & Categorías \\
\hline & \\
\hline $1,00 \geq 2,60$ & Baja \\
$2,61 \geq 3,40$ & Media \\
$3,41 \geq 5,00$ & Alta \\
\hline
\end{tabular}

Fuente: Pinto, Castro y Paz (2019)

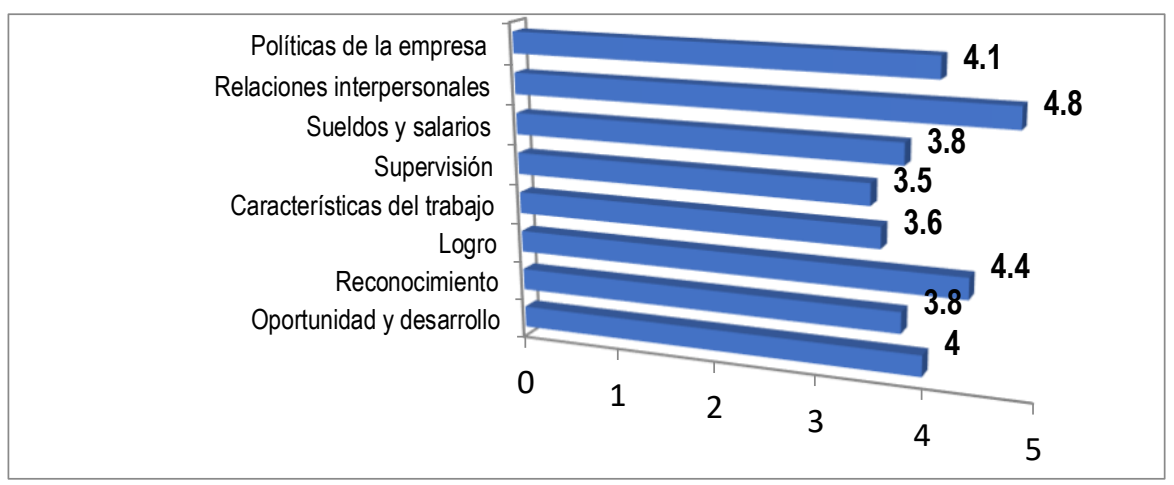

Fuente: Pinto, Castro y Paz (2019)

En relación a los factores extrínsecos de la satisfacción laboral en la universidad de La Guajira, en la tabla 1 se muestran los datos del indicador oportunidad y desarrollo, en el cual el 53\% de los empleados de carrera administrativa manifestaron que la universidad siempre les da oportunidad de crecimiento profesional en su área de trabajo; un 27\% casi siempre, $17 \%$ aduce que algunas veces y en menor porcentaje 3\% manifestó que casi nunca la universidad le da la oportunidad de crecimiento profesional. Por otra parte, el 57\% opina que siempre hay planes de ascenso en la universidad; 19\% de los encuestados indicaron que casi siempre, un $15 \%$ de los mismos manifiestan que a veces, y el menor valor porcentual se adjudicó a un 3\% se oponen a que casi nunca existen planes de ascenso en la universidad. Por consiguiente, el 49\% planteo que algunas veces reciben adiestramiento cuando hay la oportunidad de ocupar un cargo de mayor nivel; 20\% sienten que siempre, el $17 \%$ casi nunca, en tanto el $14 \%$ manifiesta que casi siempre reciben adiestramiento en el caso de existir la oportunidad de ocupar un cargo de mayor nivel en la institución. 
Tabla 4. Factores extrínsecos de la satisfacción laboral

\begin{tabular}{|c|c|c|c|c|c|c|c|c|c|c|c|c|c|c|}
\hline \multirow{3}{*}{ Indicadores } & \multicolumn{14}{|c|}{ Dimensión: Factores extrínsecos de la satisfacción laboral } \\
\hline & \multirow{2}{*}{$\begin{array}{c}\text { Alterna } \\
\text { tiva de } \\
\text { Resp. } \\
\text { Ítems }\end{array}$} & \multicolumn{2}{|c|}{$S$} & \multicolumn{2}{|c|}{ CS } & \multicolumn{2}{|c|}{$\mathrm{AV}$} & \multicolumn{2}{|c|}{$\mathrm{CN}$} & \multicolumn{2}{|c|}{$\mathrm{N}$} & \multicolumn{3}{|c|}{ Promedios } \\
\hline & & $\mathrm{Fa}$ & $\mathrm{Fr} \%$ & $\mathrm{Fa}$ & $\mathrm{Fr} \%$ & $\mathrm{Fa}$ & $\mathrm{Fr} \%$ & $\mathrm{Fa}$ & $\mathrm{Fr} \%$ & $\mathrm{Fa}$ & $\mathrm{Fr} \%$ & Item & Ind. & Dim. \\
\hline \multirow{3}{*}{$\begin{array}{l}\text { Oportunidad y } \\
\text { desarrollo }\end{array}$} & 1 & 31 & $53 \%$ & 16 & $27 \%$ & 10 & $17 \%$ & 2 & $3 \%$ & 0 & $0 \%$ & 4.29 & & \\
\hline & 2 & 34 & $57 \%$ & 11 & $19 \%$ & 9 & $15 \%$ & 4 & $7 \%$ & 1 & $2 \%$ & 4.24 & 4.0 & \\
\hline & 3 & 12 & $20 \%$ & 8 & $14 \%$ & 29 & $49 \%$ & 10 & $17 \%$ & 0 & $0 \%$ & 3.37 & & \\
\hline \multirow{3}{*}{ Reconocimiento } & 4 & 28 & $47 \%$ & 21 & $36 \%$ & 10 & $17 \%$ & 0 & $0 \%$ & 0 & $0 \%$ & 4.31 & & \\
\hline & 5 & 25 & $42 \%$ & 29 & $49 \%$ & 5 & $9 \%$ & 0 & $0 \%$ & 0 & $0 \%$ & 4.34 & 3.8 & 4.0 \\
\hline & 6 & 6 & $10 \%$ & 2 & $3 \%$ & 33 & $56 \%$ & 8 & $14 \%$ & 10 & $17 \%$ & 2.76 & & \\
\hline \multirow{3}{*}{ Logro } & 7 & 15 & $25 \%$ & 17 & $29 \%$ & 27 & $46 \%$ & 0 & $0 \%$ & 0 & $0 \%$ & 3.80 & & \\
\hline & 8 & 48 & $81 \%$ & 7 & $12 \%$ & 4 & $7 \%$ & 0 & $0 \%$ & 0 & $0 \%$ & 4.75 & 4.4 & \\
\hline & 9 & 41 & $70 \%$ & 12 & $20 \%$ & 6 & $10 \%$ & 0 & $0 \%$ & 0 & $0 \%$ & 4.59 & & \\
\hline \multirow{3}{*}{$\begin{array}{c}\text { Características del } \\
\text { trabajo }\end{array}$} & 10 & 36 & $61 \%$ & 10 & $17 \%$ & 9 & $15 \%$ & 4 & $7 \%$ & 0 & $0 \%$ & 4.32 & & \\
\hline & 11 & 25 & $42 \%$ & 14 & $24 \%$ & 11 & $19 \%$ & 9 & $15 \%$ & 0 & $0 \%$ & 3.93 & 3.6 & \\
\hline & 12 & 3 & $5 \%$ & 9 & $15 \%$ & 20 & $34 \%$ & 18 & $31 \%$ & 9 & $15 \%$ & 2.64 & & \\
\hline \multirow{3}{*}{ Supervisión } & 13 & 30 & $51 \%$ & 8 & $14 \%$ & 16 & $27 \%$ & 3 & $5 \%$ & 2 & $3 \%$ & 4.03 & & \\
\hline & 14 & 10 & $17 \%$ & 4 & $7 \%$ & 14 & $24 \%$ & 14 & $23 \%$ & 17 & $29 \%$ & 2.59 & 3.5 & \\
\hline & 15 & 21 & $35 \%$ & 18 & $31 \%$ & 11 & $19 \%$ & 9 & $15 \%$ & 0 & $0 \%$ & 3.86 & & \\
\hline \multirow{3}{*}{ Sueldos y salarios } & 16 & 25 & $42 \%$ & 18 & $31 \%$ & 13 & $22 \%$ & 3 & $5 \%$ & 0 & $0 \%$ & 4.10 & & \\
\hline & 17 & 27 & $46 \%$ & 13 & $22 \%$ & 14 & $24 \%$ & 5 & $8 \%$ & 0 & $0 \%$ & 4.05 & 3.8 & \\
\hline & 18 & 19 & $32 \%$ & 8 & $14 \%$ & 11 & $19 \%$ & 15 & $25 \%$ & 6 & $10 \%$ & 3.32 & & \\
\hline \multirow{3}{*}{$\begin{array}{c}\text { Relaciones } \\
\text { interpersonales }\end{array}$} & 19 & 49 & $83 \%$ & 7 & $12 \%$ & 3 & $5 \%$ & 0 & $0 \%$ & 0 & $0 \%$ & 4.78 & & \\
\hline & 20 & 52 & $88 \%$ & 5 & $8 \%$ & 1 & $2 \%$ & 1 & $2 \%$ & 0 & $0 \%$ & 4.83 & 4.8 & \\
\hline & 21 & 47 & $80 \%$ & 10 & $17 \%$ & 2 & $3 \%$ & 0 & $0 \%$ & 0 & $0 \%$ & 4.76 & & \\
\hline \multirow{3}{*}{$\begin{array}{c}\text { Políticas de la } \\
\text { empresa }\end{array}$} & 22 & 35 & $60 \%$ & 15 & $25 \%$ & 9 & $15 \%$ & 0 & $0 \%$ & 0 & $0 \%$ & 4.44 & & \\
\hline & 23 & 32 & $54 \%$ & 16 & $27 \%$ & 10 & $17 \%$ & 1 & $2 \%$ & 0 & $0 \%$ & 4.34 & 4.1 & \\
\hline & 24 & 13 & $22 \%$ & 16 & $27 \%$ & 26 & $44 \%$ & 3 & $5 \%$ & 1 & $2 \%$ & 3.63 & & \\
\hline
\end{tabular}

En el indicador reconocimiento, el 47\% de los encuestados, revelan que siempre se les reconoce el desempeño de su trabajo; un 36\% de la misma manera destacan que casi siempre se lleva cabo tal reconocimiento y el restante con un $8 \%$ de los empleados manifiestan que a veces. Dentro de este contexto, el $49 \%$ de los encuestados dice recibir casi siempre por escrito reconocimiento por su desempeño superior al deseado, un $42 \%$ indica que siempre, mientras el $8 \%$ de ellos sienten que algunas veces reciben el mencionado reconocimiento por su desempeño. En relación al 56\% refleja que a veces manifiesta descontento con el jefe inmediato cuando a otros le otorgan reconocimiento donde participo; un 17\% opina nunca, el 14\% respondió casi nunca y un 10\% expreso siempre su descontento ante la situación planteada, y el restante 3\% indican casi nunca. Con respecto, al indicador logro, el $46 \%$ de los encuestados opinan que el jefe a veces retroalimenta sobre los indicadores logrados en su desempeño; un 29\% casi siempre, un 25\% manifiesto siempre recibir retroalimentación por parte de su jefe inmediato de los indicadores alcanzados como resultado de su desempeño en la institución.

Dentro de este marco, el $81 \%$ de los empleados de carrera administrativa manifiestan que siempre se estimula la mejora del trabajo continuo para lograr la excelencia de la dependencia por parte del jefe inmediato, $12 \%$ indican que casi siempre, el restante $7 \%$ revelan que algunas veces el jefe inmediato estimula la mejora del trabajo continuo para 
lograr juntos la excelencia. Por su parte, el 69\% del personal de carrera administrativa señalaron que siempre proponen correctivos al detectar fallas para alcanzar los objetivos institucionales, un 20\% dicen casi siempre y el restante 10\% enunciaron algunas veces propone correctivos al detectar fallas para alcanzar los objetivos institucionales. En la tabla 4 acerca de la dimensión factores extrínsecos de calidad de vida laboral, el indicador características del trabajo, el 61\% opinan que siempre realiza el trabajo asignado aun cuando no sea lo que espera hacer; un $17 \%$ casi siempre realiza el trabajo asignado aun cuando no sea lo que espera hacer, $15 \%$ de ellos realiza el trabajo asignado aun cuando no sea lo que espera hacer, el restante $7 \%$ indica casi nunca realiza el trabajo asignado aun cuando no sea lo que espera hacer.

Según la información ilustrada en la tabla 4, el 42\% de los encuestados manifiesta su satisfacción por desarrollar a su jefe inmediato el trabajo asignado; 24\% de ellos opinan casi siempre, el 19\% revelan algunas veces y un 15\% indican que casi nunca manifiestan la satisfacción de desarrollar el trabajo asignado a su jefe inmediato. Por otra parte, el $34 \%$ de los empleados encuestados algunas veces rechaza el desarrollo de un trabajo cuando desconoce cómo elaborarlo; 30\% indican que casi nunca, un 15\% manifiestan casi siempre e igual valor porcentual es decir $15 \%$ que nunca genera rechazo ante esta situación. En el indicador Supervisión en el trabajo 51\% de los encuestados revelan que el jefe inmediato siempre estimula la gestión de su trabajo, 27\% de ellos manifiestan siempre, $14 \%$ opinan casi siempre, un 5\% de ellos opinan casi nunca y el restante $3 \%$ revelan nunca el jefe inmediato estimula la gestión de su trabajo.

Dentro de este contexto, se asume con un 29\% de los empleados de carrera administrativa refieren que nunca se establece controles para dirigirse al jefe inmediato en caso de dudas en la gestión laboral, un 24\% manifiesta algunas veces, así mismo un $23 \%$ casi nunca y un $17 \%$ manifiesta que siempre y el restante valor porcentual reflejado en un $7 \%$ opinan que casi siempre establece controles para dirigirse al jefe inmediato en caso de dudas en la gestión laboral. En referencia al 35\% de los encuestados revelan que el jefe inmediato siempre monitorea el desempeño de sus actividades para evaluar el cumplimiento; un 30\% dicen que casi siempre; un 19\% opinan que algunas veces y el restante con un $15 \%$ casi nunca manifiestan monitoreo por parte del jefe inmediato en el desempeño de sus actividades para evaluar el cumplimiento.

En relación al indicador sueldos y salario, el $42 \%$ el salario satisface las expectativas por su desempeño; $31 \%$ indican casi siempre, el $22 \%$ manifiesta algunas veces y el restante $5 \%$ de los empleados opinan casi nunca el salario satisface sus expectativas por su desempeño. Por otro particular, el 46\% opinan que el salario es atractivo en relación a otras instituciones de educación universitaria; 24\% dicen que algunas veces; un $22 \%$ dicen que casi siempre y el menor valor porcentual con un $8 \%$ manifiesta casi nunca su salario es atractivo en relación a otras instituciones de educación universitaria. Dentro de estas perspectivas, el 32\% de los encuestados opinan siempre que su salario les permite ahorrar para cubrir posibles eventualidades, mientras el 25\% expresan casi nunca, un 19\% de ellos aducen que algunas, $14 \%$ opinan casi siempre y contrario a ello el 10\% manifiesta que nunca el salario le permite ahorrar para cubrir posibles eventualidades.

Para el indicador relaciones interpersonales, el $83 \%$ de los encuestados mantiene siempre una armonía en su relación trabajo a pesar de que no sea reciproco; 12\% dicen casi 
siempre y el restante 5\% manifiesta a veces mantiene una armonía en su relación trabajo a pesar de que no sea reciproco. En relación a la opinión de los encuestados, donde los datos se reflejaron en la tabla 6 el $88 \%$ opinan siempre se relaciona con su grupo de trabajo para alcanzar juntos los objetivos institucionales, el $8 \%$ dice casi siempre y el 2\% respectivamente indican que algunas veces y casi nunca se relaciona con su grupo de trabajo para alcanzar juntos los objetivos institucionales. No obstante, el $80 \%$ de los empleados de carrera administrativa opina que siempre se comunica con su grupo de trabajo para planificar las actividades a realizar para alcanzar los objetivos institucionales, el 17\% revelan que casi siempre, el restante 3\% de ellos manifiestan algunas veces lleva a cabo dicha comunicación. Dentro de este contexto, en relación al indicador políticas de la empresa, el 60\% expresan que siempre se establece la asignación de la capacitación de acuerdo a las políticas institucionales; 25\% manifiesta casi siempre y el 15\% manifiesta que la empresa establece la asignación de la capacitación de acuerdo a las políticas institucionales.

Seguidamente, el 54\% de los encuetados se le informa siempre su quehacer laboral tomando en cuenta las políticas universitarias; $27 \%$ opinan que casi siempre; mientras un $17 \%$ de ellos algunas veces se lleva a cabo la mencionada información y el restante 2\% opinan casi nunca. Un 44\% de los empleados de carrera administrativa opinan que a veces se apoya la gestión del talento humano en políticas para satisfacer las necesidades; $27 \%$ manifiestan que casi siempre, un 22\% opinan que siempre, 5\% dicen que casi nunca y el restante $2 \%$ opinan que nunca se apoya la gestión de políticas para satisfacer las necesidades del talento humano. A modo general, los resultados porcentuales promedio destacan que el 4,8 destacan con muy alta presencia en las relaciones interpersonales en las universidades y dimensión con 4 indicando alta presencia.

\section{DISCUSIÓN}

Para hacer inferencia en los datos logrados de la aplicación de la encuesta a la población objeto de estudio con los concernientes a los aspectos teóricos de los autores citados, se establecen argumentos del comportamiento del objetivo de investigación, mediante el sustento de criterios semejantes o discrepantes, a partir de los aspectos planteados en el estudio. Con respecto al objetivo analizar los factores extrínsecos de la satisfacción laboral de los colaboradores de carrera administrativa de la Universidad de La Guajira, el indicador oportunidad y desarrollo, los encuestados manifestaron que la universidad siempre les da oportunidad de crecimiento profesional en su área de trabajo; existiendo planes de ascenso; al igual reciben adiestramiento cuando hay la oportunidad de ocupar un cargo de mayor nivel.

Coincidiendo con Ivancevich (2009), señalan que la satisfacción del personal es una actitud que los individuos y mantienen con respecto a sus funciones laborales. Por consiguiente, la dimensión que encamina estudiar elementos o indicadores asociados a la percepción de la persona sobre las oportunidades y desarrollo que tiene en la organización, sistema de reconocimientos, logros obtenidos, características del trabajo, mecanismos de supervisión, sistema o políticas de sueldos y salarios.

En el indicador reconocimiento, los encuestados, revelan que siempre se les reconoce el desempeño de su trabajo, recibiéndolo por escrito alcanzado con respuesta a 
desempeño superior al deseado, pero también muestra descontento con el jefe inmediato cuando a otros le otorgan reconocimiento donde participo. Admitiendo desde la postura de Maslow, citado por Bohlander, Snell y Sherman (2008), el reconocimiento, es herramienta asociada a las necesidades personales como lo son el ego o autoestima, donde el individuo internaliza el campo de trabajo, siendo una recompensa para conseguir destacarse en su entorno laboral; al igual debe estar sustentado en la autorrealización y el respeto a sí mismo.

En relación al indicador logro, los encuestados opinan que el jefe a veces retroalimenta sobre los indicadores logrados en su desempeño; recibiendo retroalimentación, lo cual los estimula a mejora el trabajo continuo para lograr la excelencia de la dependencia, y de ser necesario aplicar acciones correctivas. El logro, se asocia a la teoría de Ivancevich (2009), siendo el deseo que siente una persona de alcanzar objetivos claros, auto determinado y moderadamente complejo, con retroalimentación, basado en la consecución de los objetivos autodeterminados.

En la característica del trabajo, opinan los encuestados que siempre realizan el trabajo asignado aun cuando no sea lo que esperan hacer; manifestando su satisfacción por desarrollar a su jefe inmediato el trabajo, pero al igual rechazando el desarrollo de un trabajo cuando desconoce cómo elaborarlo. Mostrando semejanzas la postura de Ivancevich (2009) al mostrar que las características del trabajo, está referida con la satisfacción del empleado en relación a la naturaleza de la labor que realiza.

En el indicador supervisión en el trabajo los encuestados revelan que el jefe inmediato siempre estimula la gestión de su trabajo, donde nunca se establece controles para dirigirse al jefe inmediato en caso de dudas en la gestión laboral, sin embargo, monitorea el desempeño de sus actividades para evaluar el cumplimiento de las actividades asignadas. Coincidiendo con la teoría de los autores Chuder y Sherman (2007), sostienen que el papel del supervisor en la institución es de vital importancia, donde todo lo que se haga depende principalmente de él, al hacerse responsable de la unidad, por consiguiente, el supervisor sirve de eslabón o de enlace e influye en su personal estimulándolo, promoviendo buenas relaciones y sirviendo de paradigma ante todos sus empleados.

En relación al indicador sueldos y salario, los encuestados destacaron que el salario satisface las expectativas por su desempeño; siendo atractivo en relación a otras instituciones de educación universitaria permitiéndoles ahorrar para cubrir posibles eventualidades. Por esta razón, muestra aspectos coincidentes con Chiavenato (2011) expone que el salario es un elemento motivador, que sustenta la calidad de vida de un individuo en el contexto social, estando relacionado con el trabajo. En las organizaciones, el salario es indicador asociado tanto a un costo como a una inversión, por ello viene hacer el costo del producto o del servicio final e inversión, representando el dinero aplicado a un factor productivo (el trabajo) en un intento por obtener un rendimiento mayor al corto o mediano plazo. Agrega el autor, que las estructuras de salarios, deben están sustentadas a criterios equitativos y justos, teniendo en cuenta dos aspectos importantes, el primero, los salarios deben relacionarse con los puestos de la organización, a fin de tener un equilibrio interno de la estructura salariar de los colaboradores de ella; en segundo los salarios con respecto a empresas con naturaleza 
similar a ella en el mercado, a fin de tener una referencia de equilibrio externo en los salarios devengados por los colaboradores. Por otro lado, Castillo (2008) sobre el salario señala que es la cantidad de dinero que pacta en el contrato de trabajo y es cancelado de manera ordinaria como resultado del trabajo al colaborador, sin la adición de otros elementos que lo integran.

En relación al indicador relaciones interpersonales, los encuestados mantienen siempre una armonía en su relación trabajo a pesar de que no sea reciproco; ya que se relaciona con su grupo de trabajo para alcanzar juntos los objetivos institucionales, conllevando a una comunicación para planificar las actividades a realizar para alcanzarlos. Davis y Newstron (2008), señalan que son las interacciones entre compañeros de trabajo o individuos que comparten actividades en una misma organización.

En relación al indicador políticas de la empresa, los encuestados expresaron que la institución objeto de estudio asignación la capacitación de acuerdo a las políticas institucionales; además de informar quehacer laboral tomando en cuenta las políticas universitarias; para apoyar la gestión del talento humano y con ello satisfacer las necesidades; Davis y Newstron (2008), la definen como las conductas intencionadas diseñadas para incrementar o proteger la influencia e intereses personales de un individuo, en este caso cuando la institución considera a su personal, toma en cuenta como política la capacitación y el crecimiento tanto personal como profesional de sus empleados, para que obtengan el conocimiento y habilidades, desarrollando así sus actitudes en beneficio de su trabajo.

\section{CONCLUSIONES}

Durante el quehacer del proceso investigativo, resulta fundamental hacer inferencia aquellos aspectos que a manera de opinión de los encuestados facilitaron conocer el comportamiento de la variable en la institución objeto de estudio. De esta forma, a continuación se mencionan las generalidades percibida de la opinión de los encuestados, de acuerdo al objetivo de investigación planteado, análisis de los factores extrínsecos de satisfacción laboral de los empleados de carrera administrativa de la Universidad de La Guajira, en este particular las características del trabajo se muestra que los empleados encuestados admitieron realizar el desempeño de la labor, aun cuando no corresponde a lo que espera hacer; y posteriormente mostrar su satisfacción ante el jefe inmediato, quien estimula a obtener un trabajo de excelencia; pero también los colaboradores revelaron rechazar la asignación de trabajo que desconoce cómo elaborar.

Por otro lado, señalaron los encuestados que no se encuentran establecidos los controles para dirigirse al jefe inmediato, pero si los indicadores para monitorear el desempeño y evaluar el cumplimiento de las asignaciones como empleado, el cual se refleja en la compensación salarial que satisface sus expectativas siendo atractivo en comparación con otras instituciones Universitarias, permitiendo ahorrar. Dentro de este contexto, sobre las relaciones interpersonales, existe armonía en el trabajo, conllevando a ejercer un trabajo en conjunto para obtener los objetivos institucionales y planificar las actividades a realizar; además de explicar las políticas institucionales para asignar la capacitación, a fin de buscar la satisfacción de las necesidades profesionales e institucionales. 


\section{REFERENCIAS}

Arias, F (2016). El Proyecto de Investigación. Venezuela. Editorial Episteme. Séptima edición.

Bernal, C (2010). Metodología de la Investigación para la Administración y Economía. Santa Fe de Bogotá D. C. Colombia. Editorial Pearson. Tercera edición

Bohlander, G Snell, S y Sherman, A (2008). Administración de los Recursos Humanos. México. Editorial Thompson. Décimo cuarta edición.

Briceño, L., Ruiz, M., Villalba, V., Castro, A., Vidal, C. y Severiche, C. (2019). Elementos de la gerencia del siglo XXI: Una fundamentación desde la bioética y el liderazgo. En E. Martínez, A. Antúnez, J. Luna, V. Meriño, C. Martínez, I. Rincón (Comp), Gestión del conocimiento perspectiva multidisciplinaria. Vol. 12 (pp. 277-298). Zulia, Venezuela: Fondo Editorial Universitario de la Universidad Nacional Experimental Sur del Lago Jesús María.

Cantón Mayo, l., \& Téllez Martínez, S. (2016). La satisfacción laboral y profesional de los profesores. Revista Lasallista de Investigación, 73 (1), 214-226. http://www.redalyc. org/comocitar.oa?id=69545978019

Castillo, J (2008) Administración de personal. Un enfoque hacia la calidad de Vida. Colombia. Eco ediciones

Castro Alfaro, A., \& Anturi Santos, R. (2015). El análisis técnico y fundamental en un contexto de globalización: Bancolombia. Aglala, 6(1), 1-37.

Castro, A., Hoyos, A., Londoño, M. y Mercado, L. (2017). Estudio de marketing para medir la viabilidad comercial de una agencia publicitaria enfocada a pymes en Palmira-Valle del Cauca. Aglala, 8(1), 1-19

Chiang, M., Martín, M. J., \& Núñez, A. (2011). Relaciones entre el clima organizacional y la satisfacción laboral. Una comparación entre hospitales públicos de alta y baja complejidad. Revista Salud de los Trabajadores, 19(1), 05-16 http://www.redalyc.org/articulo.oa?id=375839297002

Chiavenato, I (2011) Administración de Recursos Humanos. El capital humano de las organizaciones. México: McGraw Hill.

Chruden, H y Sherman, A (2007) Administración de Personal. Colombia. Editorial Grupo Continental.

Código Sustantivo del Trabajo. https://legislacion.vlex.com.co/vid/codigo-sustantivo-trabajo42845853

Davis, K. y Newstrom, J. (2008). Comportamiento Humano en el trabajo. México: McGraw Hill.

Gibson, J; Ivancevich, J; Donnelly, J \& Konopaske, R (2013) Organizaciones. Comportamiento, Estructura \& Procesos. México: MacGraw Hill.

Harris, J., Franco, F. \& Paz, A. (2014). Compromiso organizacional: Estímulo del éxito del personal docente en las universidades privadas, Revista Económicas CUC, 35 (1), 91-106. Recuperado de https://revistascientificas.cuc.edu.co/economicascuc/article/view/227

Hernández, R. Fernández, C. Baptista, P. (2014). Metodología de la Investigación. México: Mc Graw Hill.

Hernández Royett, J., \& Castillo Osorio, B. (2015). Desafíos y responsabilidades de la profesión contable frente a la contabilidad ambiental. Aglala, 6(1), 164-182. doi: 10.22519/22157360.754

Hernández Royett, J., Franco, D., Canabal Guzmán, J., Sánchez Otero, M., \& D’Andreis Zapata, A. (2017). La auditoría ambiental, una atribución de las funciones del revisor fiscal. Aglala, 8(1), 219242. https://doi.org/https://doi.org/10.22519/22157360.1033

Gómez-Romo, M., López-Gomez, A., \& Carvajal-Larenas, R. (2017). La Responsabilidad Social en las PyMEs del Sector Calzado en Ecuador. Panorama Económico, 25(2), 195-208. https://doi.org/10.32997/2463-0470-vol.25-num.2-2017-2074

Ivancevich, J (2009) Administración de los Recursos Humanos. México: McGraw-Hill.

Koontz, H, Weihrich, H y Cannice, M. (2012) Administración. Una perspectiva global y empresarial. México: McGraw-Hill.

Ley 50 (1990) Reforma del Código Sustantivo del Trabajo. http://www.alcaldiabogota.gov.co/sisjur/normas/Norma1.jsp?i=281

Lozano, J (2014) Investigación Aplicada: Definición, Propiedad Intelectual e Industria. Revista CIENCIAMÉR/CA 3, 34-39. http://www.uti.edu.ec/antiguo/index.php/component/k2/item/554volumen3-cap6.html

Marquina, C (2013) Satisfacción laboral y compromiso organizacional en empleados de una universidad privada de Lima. Revista Científica de Ciencias de la Salud, 6(6). http://revistascientificas.upeu.edu.pe/index.php/rc salud/article/view/188 
Méndez, C. (2013). Metodología: diseño y desarrollo del proceso de investigación con énfasis en ciencias empresariales. México: Editorial Limusa.

Núñez, M; Salom, J; Paz, A; Rosales, V (2012) Responsabilidad Social Universitaria: Enfoque de Gestión Ética Compartidar Revista Opción, 28(69). http://www.redalyc.org/html/310/31025702007/

Pérez Vilar, P., \& Azzollini, S. (2013). Liderazgo, equipos y grupos de trabajo: su relación con la satisfacción laboral. Revista de Psicología (PUCP), 37(1), 151-169.

Peralta, Enrique. (2016). Teoría general de los sistemas aplicada a modelos de gestión. Aglala, 7(1), 122-146. https://dialnet.unirioja.es/servlet/articulo?codigo $=6832738$

Pinto, E., Brito, C., \& Mendoza, C. (2016). Equidad económica dimensión de la calidad de vida laboral en la universidad de la Guajira. Desarrollo Gerencial, 8(2), 124-143. https://doi.org/10.17081/dege.8.2.2566

Robbins, S. (2012). Comportamiento organizacional. Teoría y Práctica. México: Pearson

Rodríguez, Daysi; Núñez, Linda y Cáceres, Alfonso (2010) Estudio Comparativo de la Satisfacción Laboral universitaria en el núcleo de Anzoátegui de la Universidad de Oriente. Revista Investigación y Postgrado, 25(1), 63-80. http://www.scielo.org.ve/scielo.php?.pid=\$1316$0872010000100004 \&$ script $=$ sci abstract

Romero, Z., Navas, M., Londoño, E., Escobar, A. (2016). Responsabilidad Social: Enfoques y Perspectivas. Cartagena: Universidad Libre de Colombia. Recuperado de: http://www.unilibre.edu.co/cartagena/images/investigacion/libros/RESPONSABILIDAD-SOCIALCON-AUTORES 1.pdf

Tamayo y Tamayo, M. (2009). Metodología formal de la investigación. México: Editorial Limusa.

Vargas Cordero, Z. (2009). La investigación aplicada: una forma de conocer las realidades con evidencia científica. Educación, 33(1), 155-165. Disponible en: http://www. redalyc.org/articulo.oa?id=44015082010 